Lovreglio R., Gonzalez V., Amor R., Spearpoint M., Thomas J., Trotter M., and Sacks R.(2017). "The Need for Enhancing Earthquake Evacuee Safety Using Virtual Reality Serious Games”. In: LC3 2017: Volume I Proceedings of the Joint Conference on Computing in Construction (JC3), July 4-7, 2017, Heraklion, Greece, pp. 381-389. DOI: https://doi.org/10.24928/JC3-2017/0058.

\title{
THE NEED FOR ENHANCING EARTHQUAKE EVACUEE SAFETY BY USING VIRTUAL REALITY SERIOUS GAMES
}

\author{
Ruggiero Lovreglio', Vicente Gonzalez ${ }^{2}$, Robert Amor ${ }^{3}$, Michael Spearpoint ${ }^{4}$, \\ Jared Thomas ${ }^{5}$, Margaret Trotter ${ }^{6}$, and Rafael Sacks ${ }^{7}$
}

\begin{abstract}
Enhancing evacuee safety is a key factor in reducing the number of injuries and deaths that result from earthquakes. This can be achieved by designing safer buildings taking into account behavioral factors and by training occupants. However, traditional approaches such as evacuation drills cannot be used in buildings in which occupants cannot easily leave the building (e.g. hospitals). In addition, these traditional approaches may not provide the intended learning outcomes and do not allow for the investigation of the impact of multiple evacuation factors on behavior. Virtual Reality (VR) and Serious Games (SG), i.e. games having education (in its various forms) as the primary goal, represent novel and effective alternatives to overcome the limitations of traditional approaches.

We discuss the advantages and limitations of using VR SGs to investigate how building occupants behave during earthquake evacuations and to train building occupants to cope with such emergencies. We explore the key design components to develop the VR SG framework namely (a) what features constitute an earthquake event; (b) which types of buildings can be selected and how they should be represented within the VR environment; (c) how damage to the building is to be determined and represented; (d) what factors need to be included for agent behavior in earthquakes; and (e) what level of interaction should there be between agents and the human players. We highlight the important aspects that need to be addressed in further research to effectively develop VR SG tools for earthquake evacuation training.
\end{abstract}

Keywords: Serious Game, Virtual Reality, Earthquake Evacuation, Human Behavior, Occupant Training

\section{INTRODUCTION}

Drop, cover and hold during an earthquake and evacuating buildings after it represent the main response strategies to cope with such a threat. Enhancing evacuee safety is a key factor to reduce the number of injuries and deaths and this goal can be achieved by using two risk-reduction approaches. The first one consists of using a 'behavioral design' approach to define riskreduction interventions in new and existing buildings (Bernardini, D’Orazio, et al. 2016). This strategy relies on the knowledge of behavioral paradigms concerning how building occupants behave in earthquake evacuations. The second approach consists of training building occupants in order to enhance their earthquake evacuation preparedness by providing them with the information about how to be ready for an earthquake and how to behave during and after an earthquake.

\footnotetext{
${ }^{1}$ Research Fellow, University of Auckland, Auckland, New Zealand, r.lovreglio@auckland.ac.nz

${ }^{2}$ Senior Lecturer, University of Auckland, Auckland, New Zealand, v.gonzalez@auckland.ac.nz

${ }^{3}$ Professor, University of Auckland, Auckland, New Zealand, trebor@cs.auckland.ac.nz

${ }^{4}$ Associate Professor, University of Canterbury, Christchurch, New Zealand, michael.spearpoint@canterbury.ac.nz

${ }^{5}$ Research Manager, Opus, Wellington, New Zealand, jared.thomas@opus.co.nz

${ }^{6}$ Senior Researcher, Opus, Wellington, New Zealand, margaret.trotter@opus.co.nz

${ }^{7}$ Associate Professor, Technion, Haifa, Israel, cvsacks@techunix.technion.ac.il
} 
Different approaches can be used to investigate evacuation behavior and train building occupants. Evacuation drills are the most used traditional approach to investigate evacuation behavior and train building occupants. The main limitation of evacuation drills is the difference between the real world emergency and the simulated emergency. In earthquake scenarios one of these differences is the impossibility of taking into account the building damage and its impact on human behaviour. Moreover, evacuation drills might trigger evacuation behaviors that are different from those observed in real earthquake evacuations (Bernardini, D'Orazio, et al. 2016). From a pedagogical point of view, it is difficult to ensure that evacuation drill participants are subject to effective training. In fact, often evacuation drill participants do not receive feedback allowing them to assess their evacuation choices (Gwynne et al. 2016; Gwynne et al. 2017).

Nowadays Virtual Reality (VR) and Serious Games (SG) represent novel and effective alternatives to overcome the limitations of traditional approaches (Chittaro \& Ranon 2009). On the one hand, VR technologies (i.e. tools to immerse users in a computer generated synthetic environment) have been proved to be a valuable alternative to investigate several behaviors in fire evacuation such as system perception (Ronchi et al. 2016; Olander et al. 2017), premovement behavior, wayfinding, exit choice (Kobes et al. 2010; Lovreglio et al. 2016) and navigation interactions (Lovreglio et al. 2015a). This emerging technology allows participants to be exposed to more realistic evacuation scenarios by allowing the representation of several threats such as fire and earthquake building damage. On the other hand, SG concepts (i.e. games having education as the primary goal rather than pure entertainment) have been used over recent decades to train people to cope with different fire emergency issues (Williams-Bell et al. 2015) and several evacuation scenarios (Rüppel \& Schatz 2011; Chittaro \& Buttussi 2015). However, despite the advantages provided by VR and SG, applications in the space of earthquake evacuations are still rare.

The objective of this paper is to investigate the combined use of VR and SGs to identify the key components necessary to develop a laboratory to investigate human behavior in earthquake evacuation and a training tool to teach building occupants how to cope with this threat.

\section{SERIOUS GAMES AND ViRTUAL REALITY}

SGs represent a new means to investigate human behavior and to train building occupants to cope with earthquake evacuations. To date, several definitions have been provided and discussed for SGs. For instance, Michal and Chen (2006, p.17) define a SG as "a game in which education (in its various forms) is the primary goal, rather than entertainment" highlighting that education and entertainment are not in conflict but overlap enhancing the outcomes of the experience for the players.

SGs have been tested for training purposes in fire emergencies (Williams-Bell et al. 2015), and for enhancing players' earthquake preparedness. For instance, Tanes \& Cho (2013) investigated the possibility of preparing people to take precautions against earthquakes by using the online game entitled 'Beat the Quake!' developed by the Earthquake Country Alliance.

Despite the increasing number of SG applications for safety training, the advantages and disadvantages of using such new tools to train building occupants is still under investigation. The main advantage is that serious games can dramatically improve the realism of the learning experience. Features such as debris and unexpectedly blocked exit pathways can easily be included in virtual environments without exposing players to any risk. VR simulations have similarly been used for construction safety training research, where participants can experience hazardous scenarios without risk (Sacks et al. 2013). Another important advantage is the high control of the evacuation scenarios. Different evacuation scenarios can be designed and used to enhance the training outcomes of the game (Rüppel \& Schatz 2011). However, there is still the 
need for more longitudinal experimental studies to assess the long-term effects of training using serious games (Chittaro \& Buttussi 2015).

Besides the training purpose of the SGs, these tools also provide the possibility of investigating players' behaviors (Rüppel \& Schatz 2011). SGs allow the assessment of players' reactions to different stimuli and their decision-making. For instance, simulating the earthquake damage in the virtual environment can allow the assessment of a player's reaction to different evacuation conditions (e.g. different level of damage and different visibility conditions). Therefore, a SG platform can be seen as a virtual laboratory with higher experimental control than 'traditional' evacuation tools. The validity of such behavioral data can be increased by coupling SGs with new emerging immersive VR technologies. These technologies allow the user to be immersed in a computer generated virtual or synthetic environment by using head mounted displays (HMDs) or Cave Automatic Virtual Environments (CAVEs) (Nilsson \& Kinateder 2015).

The main advantage provided by platforms coupling VR and SGs is the amount of behavioral data that can be collected during the game experience. Immersive VR technologies allow the investigation of evacuee movement like classic evacuation experiments do. However, they can also allow the investigation of evacuee viewing directions and which elements and objects the players are looking at before making their decisions (Rüppel \& Schatz 2011). This type of data, which are difficult to collect with classic evacuation experiments such as evacuation drills, can be fundamental to identify what factors are affecting evacuation behavior (Nilsson \& Johansson 2009; Lovreglio et al. 2015b).

Despite the advantages offered by VR technologies to study human behavior, there are still technological challenges and limits that need to be addressed (Nilsson \& Kinateder 2015). For instance, many HMDs do not allow users to see their own body in the virtual environment. This issue can be solved by combining HMDs with other sensors able to integrate the users' body in the virtual environment. Another common issue for HMDs and CAVEs is the limited space to track the movement of users (i.e. trackable areas). Omnidirectional treadmills or similar technologies and game controllers represent a solution to allow the navigation in virtual environments to have walkable surfaces bigger than trackable areas. Other open challenges related with immersive VR technologies are motion sickness (such as symptoms of nausea and vertigo), multisensory simulation (i.e. limited tactile and olfactory experiences), and interactivity (i.e. the need for game controllers to interact with objects in the virtual environment) (Nilsson \& Kinateder 2015).

Finally, the development of VR SGs for earthquake evacuations can raise ethical issues since players are immerse in scenarios that can generate strong emotions (i.e. fear and upset) or recall previous personal dramatic experiences (for instance, whenever a player has directly experienced an earthquake).

\section{EARTHQUAKE GAME ENVIRONMENT}

We propose the development of a VR SG prototype for earthquake evacuation to investigate how building occupants behave and to train building occupants in coping with such emergencies. This goal is achieved by addressing the key design components that have been identified through several focus groups with experts on earthquake safety. Those components are (a) what features constitute an earthquake event; (b) which types of buildings are to be selected and how should they be represented within the VR environment; (c) how damage to the building is to be determined and represented; (d) what factors need to be included to model agents' behavior in earthquakes; and (e) what level of interaction should there be between agents and the human players. 


\subsection{Earthquake Features}

The earthquake event represents the main component that needs to be defined to identify the gaming timeframe for the VR SG prototype. Earthquakes are a sudden and perceptible shaking of the earth surface and they can generally be characterized by three seismic stages: the foreshock stage, the main shock and the aftershock stage. The sequence of three stages can last several months (Jones 1994). This timeframe is definitely not compatible with the duration of a gaming experience. Therefore, in the context of the VR SG prototype, we identify the earthquake condition as the timeframe which can include at least the main shock and the time required to evacuate the building after the main shock. Considering that the probability of foreshock and aftershock events within the timeframe of the main shock is high (Bannister \& Gledhill 2012), it can be interesting to include foreshocks and aftershocks to train players on how to cope with those events, as well as to investigate their behavior. As such, the gaming timeframe is likely to be of the order of several tens of minutes at most depending on the duration of shocks and the time required to evacuate the building. This time needs to be balanced against factors like participant fatigue.

The representation of shakes in a virtual environment represents a key challenge for the development of a VR SG prototype. An earthquake is characterized by perceptible shaking while the standard immersive technologies do not allow the inclusion of physical shaking stimuli. Therefore, players can sense the earthquake by looking at its impact on the building elements and the movement of objects in the virtual environment, including receiving auditory feedback during the event. Falling objects as well as debris formations can be 'easily' represented in the virtual environment by combining particle systems and the physical engine of the selected game engine (Rüppel \& Schatz 2011).

Different solutions can be identified to include the physical shaking dimension (i.e. $4^{\text {th }}$ game dimension) in the gaming experience, namely (1) shake tables, (2) seat or couch shaking systems and (3) game controller vibration motors. Solution (1) represents the most advanced approach as it allows players to sense the shaking experience that they would experience in a real earthquake. However, despite the high level of realism, such a solution has a location constraint as it can only be placed in a laboratory and comes with associated costs. Another limitation consists of the possibility for players to get injured by falling due to a lack of balance during the gaming experience. Solution (3) is the easiest one to setup without any location constraints, however, it provides a low level of realism as the game controller shaking can only provide a basic sense of vibrations for the SG players. Solution (2) represents a good compromise between shaking tables and game controller shakers. Seats and couches can be easily transported and can generate a relatively high shaking experience.

\subsection{Building Selection and Representation}

The game location represents the second key element to define for the VR SG prototype. It is possible to select either a real building or a hypothetical building (Chittaro \& Ranon 2009). The first approach provides players with a virtual environment they can be familiar with when players are building occupants of the existing building. This approach allows the investigation of whether and at what level building occupants are familiar with the evacuation plans and the evacuation routes, and the training of building occupants to enhance their evacuation preparedness in case of an earthquake (i.e. the set of rules to follow during and after an earthquake) for a given building. The same virtual environment can be used to investigate the behavior of players who are not familiar with a given building to assess whether or not they follow the evacuation procedure as intended in the evacuation plans. However, this solution requires enough data to develop a virtual environment having features (i.e. the geometry, materials and pieces of furniture) fairly similar to the existing built environment (Rüppel \& Schatz 2011). In contrast, the second approach (the use of a hypothetical building) simplifies the 
process of creating a virtual environment since it does not require the collection of any realworld data (Chittaro \& Ranon 2009). However, the use of a hypothetical building does not provide the possibility to investigate the impact of occupants' familiarity with the building layout, which can be a key behavioral factor during building evacuations. Different solutions can be used to make players familiar with hypothetical virtual environments, e.g. letting them navigate in it before the emergency evacuation is run within the VR SG framework. Another limitation of hypothetical buildings is that they do not have any evacuation plans that can be a fundamental piece of information to define the learning outcomes of a SG. However, evacuation plans from existing buildings that are similar to the hypothetical one can be used for such a purpose or even evacuation recommendations provided by emergency regulatory agencies may be used.

Depending on the selected building (i.e. hypothetical vs existing buildings), different strategies can be used to represent the built environment and its geometry within the game structure. For instance, in the case of existing buildings, a comprehensive representation of the geometry of a building can be created by importing an existing BIM model into the game engine as discussed by Rüppel and Schatz (2011). Moreover, more detailed information that might not be included in the original BIM model (building element material, pieces of furniture, lighting conditions and evacuation systems) can be collected through inspections of the building and integrated into the BIM model. In the case of hypothetical buildings, the representation of such a building can be inspired by existing BIM models of actual or new buildings.

In either case BIM models do not need to be extremely accurate representations of the building itself, but a sufficiently realistic model that can provide an appropriate sensory experience to players in terms of a building geometry. In fact, parts of the buildings can be deleted or replaced depending on the SG goals.

A final challenge is the representation of the environment surrounding the selected building as well as the external representation of the building itself. There may be external damage and hazards that players might need to be aware of before reaching designated safe places (e.g. assembly areas).

\subsection{Damage Representation}

The representation of the earthquake damage to the building is another challenge that needs to be addressed when developing the VR SG prototype. An earthquake can impact the structural elements (i.e. components transferring the loads of the building to the ground) and nonstructural elements (i.e. components and systems that are permanently attached to structural elements but do not transfer loads) of a building and the objects in the building such as pieces of furniture. The representation of building damage can be done following two strategies, namely quantitative and qualitative.

Quantitative strategies consist of modelling all the structural elements of a building (i.e. geometry and materials) to investigate the impact of a hypothetical or recorded earthquake on these elements. Then, the damage of the non-structural elements and on the systems can be predicted by using fragility functions, which are relationships between the probability of failure of a non-structural element and the damage to the structural elements. This approach has already been used to predict the impact of an earthquake on building evacuation using different evacuation simulation approaches (Liu et al. 2015). The main limitation of the quantitative approach is that it requires advanced calculations as well as many pieces of information such as the structural and non-structural building components, earthquake location and magnitude, ground motion conditions, etc. The number of assumptions that are necessary for those calculations can be large enough to make the damage representation as reliable as a qualitative representation.

While such calculations are fundamental for simulation studies, they are not essential for the development of a SG. A realistic representation of the building damage in SGs can be created 
by using a qualitative strategy. Such a strategy is based on mimicking the damage in the virtual environment by using data from existing datasets including videos and pictures of building damage. It does not necessarily have the same impact on the outcomes of the VR SGs since building occupants are not aware of how the building would behave in case of an earthquake. Therefore, it can be considered a valid approach as the realism of building damage should be in line with the expectations of the players. The qualitative approach lets the SG developers be free to locate the damage in 'strategic positions' of the building for specific training purposes and to investigate specific evacuation behaviors. By using the qualitative strategy, it is still possible to assume and represent realistic levels of damage for pieces of furniture in the building.

\subsection{Agent Behavior and Player-Agent Interactions}

A VR SG prototype can provide the possibility to investigate how player behavior is influenced by other evacuees. This can be done by using multi-player games, agent modelling or a combination of those two solutions. The first solution allows several players to live and interact in the same virtual environment. However, the number of players can be strongly limited by the VR hardware, for instance the number of available HMDs and the graphic card performance. The second solution does not have this limitation as many evacuees can be modelled as agents. Representing evacuation agents in a virtual environment is a further challenge for the development of a VR SG prototype. These agents should behave following existing data on human behavior in earthquake evacuations. Qualitative data (e.g. behavioral statements) and quantitative data (e.g. walking speeds and evacuation flows) on earthquake evacuation behavior can be identified in the existing literature. These data have been collected using different techniques, namely video analysis (Bernardini, Quagliarini, et al. 2016); interviews and questionnaires (Lindell et al. 2016); direct observations during an earthquake (Alexander 1990); and evacuation drills (Johnston et al. 2011). Despite the existing lack of a comprehensive literature review on human behavior in earthquake evacuations, several key behavioral statements and qualitative data can be identified and used to program agent behavior during an earthquake. A summary of some general behavioral assumptions to take into account to define agent behaviors is presented below

1. Behavioral responses and occupant's stress are strongly influenced by the earthquake perception and floor shaking (Bernardini, Quagliarini, et al. 2016).

2. Evacuees make rational decisions depending on the information available, the time available to process such information and their level of stress and fright (i.e. panic behavior is a misassumption) (Alexander 2010).

3. Social interactions, such as helping behavior and group behavior, are common during earthquake evacuations (Bernardini, Quagliarini, et al. 2016).

In reaction to the building damage, the behavior of agents should be realistic enough but it does not need to represent the actual behavior that the occupants would have in a specific scenario. In fact, different agent responses can be included in the VR SG prototype to investigate how players can be affected by the social interactions with the agents.

\section{BEHAVIORAL AND LEARNING OUTCOMES}

One crucial step for the development of VR SGs for earthquake evacuations is the definition of their goals (or outcomes) since a SG can provide leaning outcomes to players as well as behavioral outcomes for the SG developers by observing player behavior.

The learning outcome in case of earthquake evacuation aims to provide players with knowledge on how to respond during the main shock and how to evacuate safely from a building damaged by an earthquake. This can be done by identifying the inappropriate behaviors that evacuees can have in earthquake evacuations to correct them. Once these behaviors have been 
identified a fundamental question is: how can feedback be provided to the players? Several solutions have been used so far in existing SG for evacuation training. For instance, Chittaro and Buttusi (2015) use several non-verbal vocal sounds of human distress, sounds of breaking bones, blood squirts, simulation of temporary blindness, tinnitus or dizziness and a life bar (i.e. a bar showing the health of the virtual player in the game) to highlight the severity of the consequence of the unsafe actions taken by the SG players coupled with a cause of death message and a behavioural recommendation. Less extreme approaches include the use of 'points' for correct decisions and the loss of points or time penalties for incorrect decisions (Tanes \& Cho 2013). In many of these 'death' scenarios players are taken back to the point before their incorrect decision and allowed to replay from that point. The former, more extreme methods, discussed above have the advantage of adding realism, potentially eliciting a motivational response some degrees closer to that they would experience during an actual earthquake; however, there are ethical considerations in a country (such as New Zealand) where a portion of the population has been affected by a major earthquake, which may preclude their use in favour of lower fidelity options.

Finally, VR SGs provide the possibility of investigating how players behave and the factors influencing their decision-making. SGs can be used either to investigate how they respond during the main shock (e.g. drop-cover-hold vs starting evacuation) and after the shaking phase (e.g. using the closest exit vs the most familiar exit, helping other evacuees, etc.) and the factors that affect the observed behaviors. VR SGs provide high experimental control as players can be exposed to very different emergency scenarios. The main advantage of using these tools is to record the players' reaction to very extreme events (e.g. falling objects), which could not be investigated using traditional experimental tools because of ethical issues. Additionally, VR SGs can provide much better insights into player motivations behind their decision-making processes in real-time, through techniques like verbal protocol analysis (Banks et al. 2014). However, as for VR experiments, the main question that can be raised is about the ecological validity of the behavioral data collected using VR SGs (i.e. how close are the behaviors observed in a SG to those observed in real earthquakes?). Similarly to VR experiments, it is reasonable to assume that the behavioral data collected using VR SGs are equivalent to those collected using classic laboratory experiment and that VR SGs can represent one of the best compromises between experimental control and ecological validity (Nilsson \& Kinateder 2015).

It is clear that there is a consistent difference between fire and earthquake conditions. In many real fire evacuations, it is quite possible that most evacuees do not even see fire and smoke. Therefore, the gap of realism between real fire evacuations and 'classic' fire drills can be almost insignificant. In contrast, in earthquake evacuations everyone can perceive the threat, which is always missing in earthquake drills. Therefore, there is a significant gap between real evacuations and earthquake drills that could be reduced in future using VR SGs.

Finally, future studies need to answer several questions that are related with the selected behavioral and learning outcomes: How real do the physical and social environment need to be (i.e. building and damage representation and agents' behavior)? What types of learning outcomes are suitable to setup a credible and practical SG? What sort of earthquakes stages should be considered when drafting the storyline of SGs? How can we effectively measure behavioral impacts and learning and research goals?

\section{CONCLUSIONS}

VR SGs have both advantages and limitations for investigating human behavior during earthquake evacuations (i.e. a behavioral outcome) and for training building occupants to deal with such emergencies (i.e. a learning outcome). The design components for development of a VR SG prototype for earthquake evacuation include earthquake features; building selection and 
representation; damage representation; agent behavior and interactions; and behavioral learning outcomes.

\section{ACKNOWLEDGMENTS}

This research has been funded by the MBIE-Natural Hazards Research Platform (New Zealand), Grant Number: C05X0907.

\section{REFERENCES}

Alexander, D., 1990. Behavior During Earthquakes: A Southern Italian Example. International Journal of Mass Emergencies and Disasters, 8(1), pp.5-29.

Alexander, D.E., 2010. News reporting of the January 12, 2010, Haiti earthquake: the role of common misconceptions. Journal of Emergency Management, 8(6), pp.15-27.

Banks, V.A., Stanton, N.A. \& Harvey, C., 2014. What the drivers do and do not tell you: using verbal protocol analysis to investigate driver behaviour in emergency situations. Ergonomics, 57(3), pp.332-42.

Bannister, S. \& Gledhill, K., 2012. Evolution of the 2010-2012 Canterbury earthquake sequence. New Zealand Journal of Geology and Geophysics, 55(3), pp.295-304.

Bernardini, G., D’Orazio, M. \& Quagliarini, E., 2016. Towards a "behavioural design” approach for seismic risk reduction strategies of buildings and their environment. Safety Science, 86, pp.273-294.

Bernardini, G., Quagliarini, E. \& D’Orazio, M., 2016. Towards creating a combined database for earthquake pedestrians' evacuation models. Safety Science, 82, pp.77-94.

Chittaro, L. \& Buttussi, F., 2015. Assessing Knowledge Retention of an Immersive Serious Game vs. a Traditional Education Method in Aviation Safety. IEEE transactions on visualization and computer graphics, 21(4), pp.529-38.

Chittaro, L. \& Ranon, R., 2009. Serious Games for Training Occupants of a Building in Personal Fire Safety Skills. In 2009 Conference in Games and Virtual Worlds for Serious Applications. IEEE. Available at: http://ieeexplore.ieee.org/document/5116556/ [Accessed January 19, 2017].

Gwynne, S.M.V. et al., 2016. Pros and Cons of Egress Drills. In Interflam 2016. London, UK.

Gwynne, S.M.V. et al., 2017. Enhancing Egress Drills: Preparation and Assessment of Evacuee Performance. Fire and Materials, to appear.

Johnston, D. et al., 2011. Preparing Schools for Future Earthquakes in New Zealand: Lessons from an Evaluation of a Wellington School Exercise. Australian Journal of Emergency Management, 26(1), p.24.

Jones, L.M., 1994. Foreshocks, aftershocks, and earthquake probabilities: Accounting for the landers earthquake. Bulletin of the Seismological Society of America, 84(3), pp.892-899.

Kobes, M. et al., 2010. Exit choice, (pre-)movement time and (pre-)evacuation behaviour in hotel fire evacuation - Behavioural analysis and validation of the use of serious gaming in experimental research. Procedia Engineering, 3, pp.37-51.

Lindell, M.K. et al., 2016. Immediate behavioural responses to earthquakes in Christchurch, New Zealand, and Hitachi, Japan. Disasters, 40(1), pp.85-111.

Liu, Z. et al., 2015. Agent-Based Simulation of Building Evacuation after an Earthquake: Coupling Human Behavior with Structural Response. Natural Hazards Review, 17(1), p.4015019.

Lovreglio, R., Fonzone, A. \& Dell'Olio, L., 2016. A Mixed Logit Model for Predicting Exit Choice During Building Evacuations. Transportation Research Part A. 
Lovreglio, R., Ronchi, E. \& Nilsson, D., 2015a. A Mixed-Ordered Approach to Investigate Correlations Among Different Affordances in Fire Evacuation. In Human Behaviour in Fire Proceedings of the 6th International Symposium.

Lovreglio, R., Ronchi, E. \& Nilsson, D., 2015b. A model of the decision-making process during pre-evacuation. Fire Safety Tecnology.

Michael, D. \& Chen, S.L., 2006. Serious games : games that educate, train and inform, Thomson Course Technology.

Nilsson, D. \& Johansson, A., 2009. Social influence during the initial phase of a fire evacuationAnalysis of evacuation experiments in a cinema theatre. Fire Safety Journal, 44(1), pp.7179.

Nilsson, D. \& Kinateder, M., 2015. Virtual Rality Experiments - The Future or a Dead End? In K. Boyce, ed. 6th international symposium Human Behaviour in Fire. Cambridge: Interscience Communications, pp. 13-22.

Olander, J. et al., 2017. Dissuasive exit signage for building fire evacuation. Applied Ergonomics, 59, pp.84-93.

Ronchi, E. et al., 2016. A Virtual Reality experiment on flashing lights at emergency exit portals for road tunnel evacuation. Fire Technology, 52(3), pp.623-647.

Rüppel, U. \& Schatz, K., 2011. Designing a BIM-based serious game for fire safety evacuation simulations. Advanced Engineering Informatics, 25(4), pp.600-611.

Sacks, R., Perlman, A. \& Barak, R., 2013. Construction safety training using immersive virtual reality. Construction Management and Economics, 31(9), pp.1005-1017.

Tanes, Z. \& Cho, H., 2013. Goal setting outcomes: Examining the role of goal interaction in influencing the experience and learning outcomes of video game play for earthquake preparedness. Computers in Human Behavior, 29(3), pp.858-869.

Williams-Bell, F.M. et al., 2015. Using Serious Games and Virtual Simulation for Training in the Fire Service: A Review. Fire Technology, 51(3), pp.553-584. 